\title{
OSCILLATORY FLUID FLOW AND HEAT TRANSFER THROUGH POROUS MEDIUM BETWEEN PARALLEL PLATES WITH INCLINED MAGNETIC FIELD, RADIATIVE HEAT FLUX AND HEAT SOURCE
}

\author{
T. MEHTA \\ Department of Mathematics, S. S. Jain Subodh College \\ Jaipur (Raj.), INDIA \\ E-mail: m.tripti24@gmail.com \\ R. MEHTA* \\ Department of Mathematics \& Statistics, Manipal University Jaipur \\ Jaipur (Raj.), INDIA \\ E-mail: ruchika.mehta1981@gmail.com \\ A. MEHTA \\ Poornima Institute of Engineering \& Technology \\ Jaipur (Raj.), INDIA \\ E-mail: mehta.anu25@gmail.com

\begin{abstract}
The aim of the paper is to investigate an oscillatory fluid flow and heat transfer through a porous medium between parallel plates in the presence of an inclined magnetic field, radiative heat flux and heat source. It is assumed that electrical conductivity of the fluid is small and the electromagnetic force produced is very small. The governing coupled equations of motion and energy are solved analytically. Numerical results for the velocity and temperature profiles, local skin friction coefficient and local Nusselt number for various values of physical parameters are discussed numerically and presented graphically.
\end{abstract}

Key words: oscillatory, inclined magnetic field, porous medium, radiation, heat source, skin-friction coefficient and Nusselt number.

\section{Introduction}

The effect of heat transfer on an oscillatory flow of an electrically conducting fluid in vertical media is encountered in a wide range of engineering areas, science and technology such as magnetohydroynamic (MHD) power generators, plasma studies, nuclear reactor, geothermal energy extraction, electromagnetic propulsion, the boundary layer control in the field of aerodynamics. It also has numerous industrial applications in molten iron flow, recovery extraction of crude oil, electrostatic precipitation, petroleum industry and polymer technology.

Nigam and Singh [1] studied heat transfer by a laminar flow between parallel plates under the action of a transverse magnetic field. Verma and Bansal [2] presented a flow of a viscous incompressible fluid between two parallel plates, one in uniform motion and the other at rest with uniform suction at the stationary plate. Soundalgekar and Bhat [3] discussed are oscillatory channel flow and heat transfer. Raptis et al. [4] considered an MHD flow between two parallel plates with heat transfer. Sharma and Sharma [5] investigated an unsteady flow and heat-transfer between two parallel plates. Bhuyan and Hazarika [6] obtained the effect of a magnetic field on a pulsatile blood flow in a porous channel. Sharma and Chaturvedi [7]

\footnotetext{
${ }^{*}$ To whom correspondence should be addressed
} 
considered an unsteady flow and heat transfer of an electrically conducting viscous incompressible fluid between two non-conducting parallel porous plates under a uniform transverse magnetic field. Ganesh and Krishnambal [8] discussed a magnetohydrodynamic flow of a viscous fluid between two parallel porous plates. Ghosh [9] presented a hydromagnetic fluctuating flow of a viscoelastic fluid in a porous channel. Sharma and Singh [10] investigated effects of variable thermal conductivity and heat source/sink on MHD flow near a stagnation point on a linearly stretching sheet. Makinde and Aziz [11] analyzed MHD mixed convection from a vertical plate embedded in a porous medium with a convective boundary condition. Adhikary and Misra [12] presented an unsteady two-dimensional hydromagnetic flow and heat transfer of a fluid. Singh [13] described a viscoelastic mixed convection MHD oscillatory flow through a porous medium filled in a vertical channel. Das [14] presented viscoelastic effects on unsteady two dimensional heat and mass transfer of a viscoelastic fluid in a porous channel with radiative heat transfer. Sandeep and Sugunamma [15] discussed the effect of an inclined magnetic field on an unsteady free convection flow of a dusty viscous fluid between two infinite flat plates filled by a porous medium. Joseph et al. [16] studied an unsteady MHD Couette flow between two infinite parallel porous plates in an inclined magnetic field with heat transfer.

The aim of the paper is to investigate an oscillatory fluid flow and heat transfer through a porous medium between parallel plates in the presence of an inclined magnetic field, radiative heat flux and heat source.

\section{Mathematical analysis}

Consider the oscillatory flow of an electrically conducting fluid and heat transfer between infinite parallel plates at a distance $d$ apart filled with a porous medium under the influence of an inclined magnetic field and heat source. The $x^{*}$ - axis is taken along the plate (when $y^{*}=0$ ) and the $y^{*}$ - axis is taken normal to the plate. As the plates are infinite in length, the velocity and temperature fields are functions of $y^{*}$ and $t^{*}$ only. Under the above assumptions and usual Boussinesq approximation, the governing equations of motion and energy are as follows

$$
\begin{aligned}
& \frac{\partial u^{*}}{\partial t^{*}}=-\frac{1}{\rho} \frac{\partial P^{*}}{\partial x^{*}}+v \frac{\partial^{2} u^{*}}{\partial y^{* 2}}+g \beta\left(T^{*}-T_{0}\right)-\frac{\sigma_{e} B_{0}{ }^{2} \sin ^{2} \varphi}{\rho} u^{*}-\frac{v}{K^{*}} u^{*}, \\
& \frac{\partial T^{*}}{\partial t^{*}}=\frac{\kappa}{\rho C_{p}} \frac{\partial^{2} T^{*}}{\partial y^{*}}-\frac{1}{\rho C_{p}} \frac{\partial q_{r}}{\partial y^{*}}+\frac{Q_{0}}{\rho C_{p}}\left(T^{*}-T_{0}\right)
\end{aligned}
$$

where $u^{*}$ is the fluid velocity in $x^{*}$ - direction, $t^{*}$ is the time, $P^{*}$ is the fluid pressure, $T^{*}$ is the fluid temperature, $v$ is the kinematic viscosity, $\beta$ is the coefficient of the thermal expansion, $g$ is acceleration due to gravity, $B_{0}$ is the strength of the magnetic field, $\sigma_{e}$ is the electric conductivity, $\rho$ is the fluid density, $\varphi(0 \leq \varphi \leq \pi)$ is the angle between velocity and strength of the magnetic field, $K^{*}$ is the permeability of porous medium, $\kappa$ is the thermal conductivity, $C_{p}$ is the specific heat at constant pressure, $q_{r}$ is the radiative heat flux in the $y^{*}$ - is direction and $Q_{0}$ is the heat generation/ absorption constant.

The boundary conditions are given by

$$
\begin{aligned}
& y^{*}=0: u^{*}=0, \quad T^{*}=T_{0} ; \\
& y^{*}=d: u^{*}=U_{0} e^{i \omega^{* *} t^{*}}, \quad T^{*}=T_{0}+\left(T_{w}-T_{0}\right) e^{i \omega^{* *} t^{*}}
\end{aligned}
$$

where $T_{0}$ and $T_{w}$ are the temperature at the plates at $y^{*}=0$ and $y^{*}=d$, respectively. 
It is assumed that the fluid is optically thin with a relative low density and radiative heat flux is according to Cogley et al. [17] and given by

$$
\frac{\partial q_{r}}{\partial y^{*}}=4 \alpha^{2}\left(T^{*}-T_{0}\right)
$$

where $\alpha$ is the mean radiation absorption coefficient.

Introducing the following dimensionless quantities

$$
\begin{aligned}
& u=\frac{u^{*}}{U_{0}}, \quad x=\frac{x^{*}}{d}, \quad y=\frac{y^{*}}{d}, \quad t=\frac{U_{0}}{d} t^{*}, \quad \omega=\frac{d}{U_{0}} \omega^{*}, \quad P=\frac{d}{\mathrm{v} \rho U_{0}} P^{*}, \\
& \theta=\frac{T^{*}-T_{0}}{T_{w}-T_{0}}, \quad \mathrm{Re}=\frac{U_{0} d}{v}, \quad \mathrm{Ha}^{2}=\frac{\sigma_{e} B_{0}^{2} d^{2}}{v \rho}, \quad \mathrm{Gr}=\frac{g \beta d^{2}\left(T_{w}-T_{0}\right)}{v U_{0}}, \\
& K^{2}=\frac{d^{2}}{K^{*}}, \quad \mathrm{Pe}=\frac{U_{0} d \rho C_{p}}{\kappa}, \quad R^{2}=\frac{4 \alpha^{2} d^{2}}{\cup \rho C_{p}}, \quad S=\frac{Q_{0} d^{2}}{\kappa},
\end{aligned}
$$

into Eqs (2.1) and (2.2), we get

$$
\begin{aligned}
& \operatorname{Re} \frac{\partial u}{\partial t}=-\frac{\partial P}{\partial x}+\frac{\partial^{2} u}{\partial y^{2}}+\operatorname{Gr} \theta-\left(M^{2}+K^{2}\right) u \\
& \operatorname{Pe} \frac{\partial \theta}{\partial t}=\frac{\partial^{2} \theta}{\partial y^{2}}-R^{2} \theta+S \theta
\end{aligned}
$$

where $u$ is the dimensionless velocity along $x$-axis, $t$ is the dimensionless time, $y$ is dimensionless coordinate axis normal to the plates, $P$ is dimensionless pressure, $\theta$ is dimensionless temperature, $\mathrm{Gr}$ is the Grashof number, Re is the Reynolds number, Ha is the Hartmann number, $K$ is the permeability parameter, Pe is the Peclet number, $R$ is radiation parameter, $S$ is the heat source parameter.

$$
M=\text { Ha } \sin \varphi .
$$

The boundary conditions are reduced to

$$
\begin{aligned}
& y=0: u=0, \quad \theta=0 ; \\
& y=1: u=e^{i \omega t}, \quad \theta=e^{i \omega t} .
\end{aligned}
$$

\section{Method of solution}

For a purely oscillatory flow, substituting

$$
-\frac{\partial P}{\partial x}=\lambda e^{i \omega t}, \quad u(y, t)=u_{0}(y) e^{i \omega t}, \quad \theta(y, t)=\theta_{0}(y) e^{i \omega t},
$$

into Eqs (2.6) and (2.7), we get 


$$
\begin{aligned}
& \frac{d^{2} u_{0}}{d y^{2}}-a_{1}^{2} u_{0}=-\lambda-\mathrm{Gr} \theta_{0}, \\
& \frac{d^{2} \theta_{0}}{d y^{2}}-a_{2}^{2} \theta_{0}=0,
\end{aligned}
$$

where $a_{1}=\sqrt{M^{2}+K^{2}+i \omega \mathrm{Re}}$ and $a_{2}=\sqrt{R^{2}-S+i \omega \mathrm{Pe}}$.

Now, the corresponding boundary conditions are

$$
\begin{aligned}
& y=0: u_{0}=0, \quad \theta_{0}=0 ; \\
& y=1: u_{0}=1, \quad \theta_{0}=1 .
\end{aligned}
$$

Equations (3.2) and (3.3) are ordinary second order coupled differential equations and are solved under the boundary conditions (3.4). Through straight forward calculations $u_{0}(y)$ and $\theta_{0}(y)$ are known. Finally, the expressions of $u(y, t)$ and $\theta(y, t)$ are known as given below

$$
\begin{aligned}
& u(y, t)=\left[\frac{\mathrm{Gr}}{\left(a_{2}^{2}-a_{1}^{2}\right)}\left\{\frac{\sinh a_{1} y}{\sinh a_{1}}-\frac{\sinh a_{2} y}{\sinh a_{2}}\right\}+\frac{\sinh a_{1} y}{\sinh a_{1}}+\frac{\lambda}{a_{1}^{2}}\left\{\frac{\sinh a_{1}(y-1)}{\sinh a_{1}}-\frac{\sinh a_{1} y}{\sinh a_{1}}+1\right\}\right] e^{i \omega t}, \\
& \theta(y, t)=\left[\frac{\sinh a_{2} y}{\sinh a_{2}}\right] e^{i \omega t} .
\end{aligned}
$$

The dimensionless stress tensor in terms of the skin-friction coefficient at both the plates are given by

$$
C_{f}=\frac{d u}{d y}=\frac{d u_{0}}{d y}+\varepsilon e^{-n t} \frac{d u_{1}}{d y} \quad \text { at } \quad y=0 \quad \text { and } \quad y=1,
$$

Hence, the skin-friction coefficient at the plate when $y=0$ is given by

$$
\left(C_{f}\right)_{0}=\left[\frac{\mathrm{Gr}}{\left(a_{2}^{2}-a_{1}^{2}\right)}\left\{\frac{a_{1}}{\sinh a_{1}}-\frac{a_{2}}{\sinh a_{2}}\right\}+\frac{a_{1}}{\sinh a_{1}}+\frac{\lambda}{a_{1}^{2}}\left\{\frac{a_{1} \cosh a_{1}}{\sinh a_{1}}-\frac{a_{1}}{\sinh a_{1}}\right\}\right] e^{i \omega t},
$$

The skin-friction coefficient at the plate when $y=1$ is given by

$$
\left(C_{f}\right)_{1}=\left[\frac{\mathrm{Gr}}{\left(a_{2}^{2}-a_{1}^{2}\right)}\left\{\frac{a_{1} \cosh a_{1}}{\sinh a_{1}}-\frac{a_{2} \cosh a_{2}}{\sinh a_{2}}\right\}+\frac{a_{1} \cosh a_{1}}{\sinh a_{1}}+\frac{\lambda}{a_{1}^{2}}\left\{\frac{a_{1}}{\sinh a_{1}}-\frac{a_{1} \cosh a_{1}}{\sinh a_{1}}\right\}\right] e^{i \omega t} .
$$

The dimensionless rate of heat transfer in terms of the Nusselt number at both the plates is given by

$$
\mathrm{Nu}=-\left(\frac{\partial \theta}{\partial y}\right)=-\left(\frac{d \theta_{0}}{d y}\right) e^{i \omega t} \quad \text { at } \quad y=0 \quad \text { and } \quad y=1 .
$$


Hence, the Nusselt number at the plate when $y=0$ is given by

$$
(\mathrm{Nu})_{0}=-\left[\frac{a_{2}}{\sinh a_{2}}\right] e^{i \omega t}
$$

The Nusselt number at the plate when $y=1$ is given by

$$
(\mathrm{Nu})_{1}=-\left[\frac{a_{2} \cosh a_{2}}{\sinh a_{2}}\right] e^{i \omega t}
$$

\section{Results and discussion}

The effects of an inclined magnetic field, radiation and heat source on an oscillatory flow of an incompressible viscous fluid through a porous medium between vertical parallel plates are investigated. Equations of momentum and energy which govern the fluid flow and heat transfer are solved by using the perturbation method. The effects of various physical parameters on the fluid velocity, temperature, skin friction coefficient and Nusselt number at the plates are observed, discussed numerically and shown through graphs.

It is observed from Figs 1 and 2 that the velocity of the fluid increases with the increase of the Grashof number or wave length. It is noted from Figs 3 to 10 that the velocity of the fluid decreases for the Reynolds number, Hartmann number, Peclet number, permeability parameter, radiation parameter, heat source parameter, frequency of the oscillation or angle between velocity and strength of the magnetic field.

It is noted from Figs 11 and 14 that the temperature of the fluid decreases due to an increase in the Peclet number, radiation parameter, heat source parameter or frequency of the oscillation.

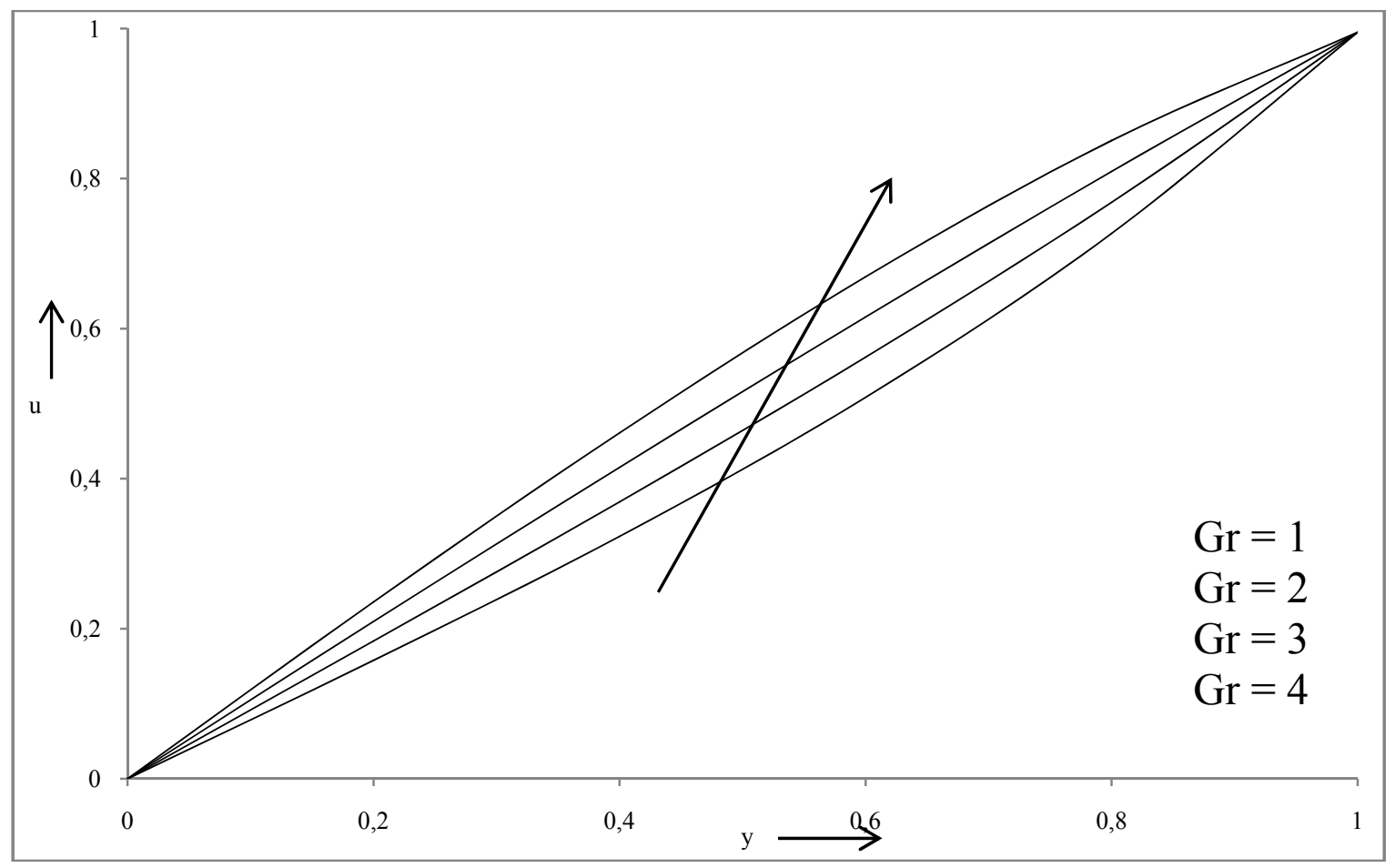

Fig.1. Velocity profiles versus $y$ for different values of $\mathrm{Gr}$ when $\lambda=0.1, \mathrm{Re}=2, \mathrm{Ha}=2, K=1, \mathrm{Pe}=0.71, R=2$, $S=5, \omega=1, \varphi=\pi / 4, g=0.1, t=0.1$. 


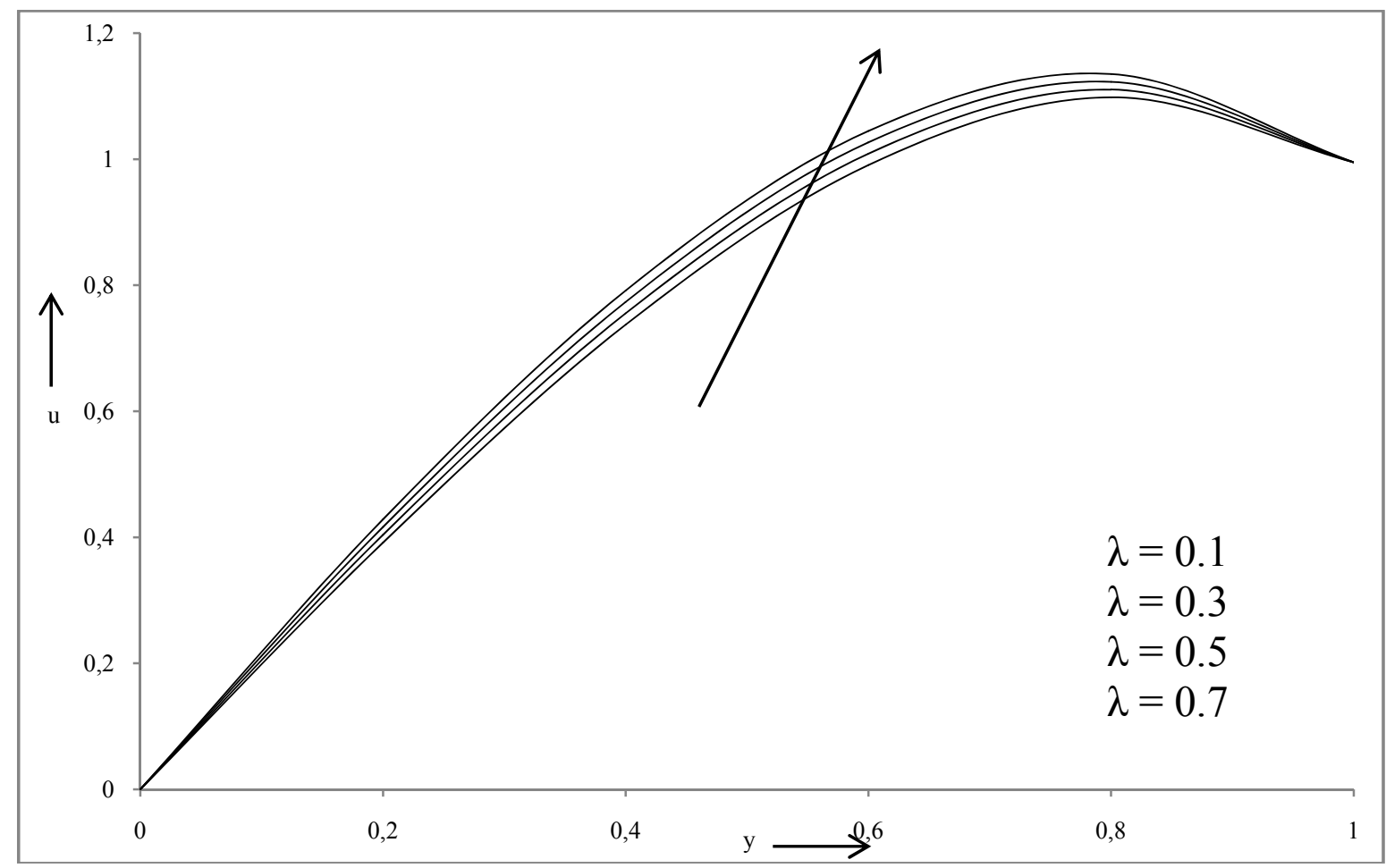

Fig.2. Velocity profiles versus $y$ for different values of $\lambda$ when $\mathrm{Gr}=10, \mathrm{Re}=2, \mathrm{Ha}=2, K=1, \mathrm{Pe}=0.71, R=2$, $S=5, \omega=1, \varphi=\pi / 4, g=0.1, t=0.1$.

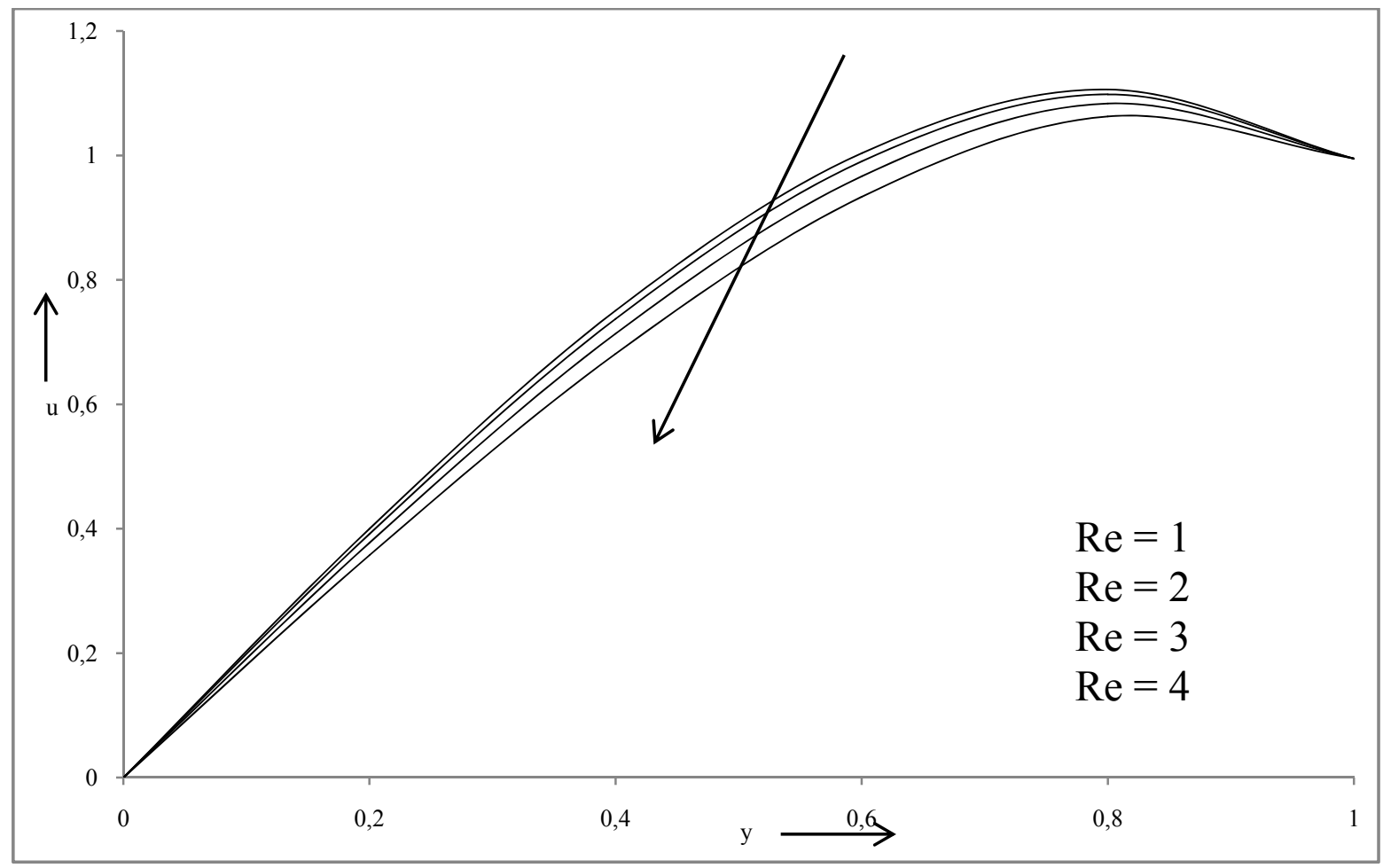

Fig.3. Velocity profiles versus $y$ for different values of $\mathrm{Re}$ when $\mathrm{Gr}=10, \lambda=0.1, \mathrm{Ha}=2, K=1, \mathrm{Pe}=0.71, R=2$, $S=5, \omega=1, \varphi=\pi / 4, g=0.1, t=0.1$. 


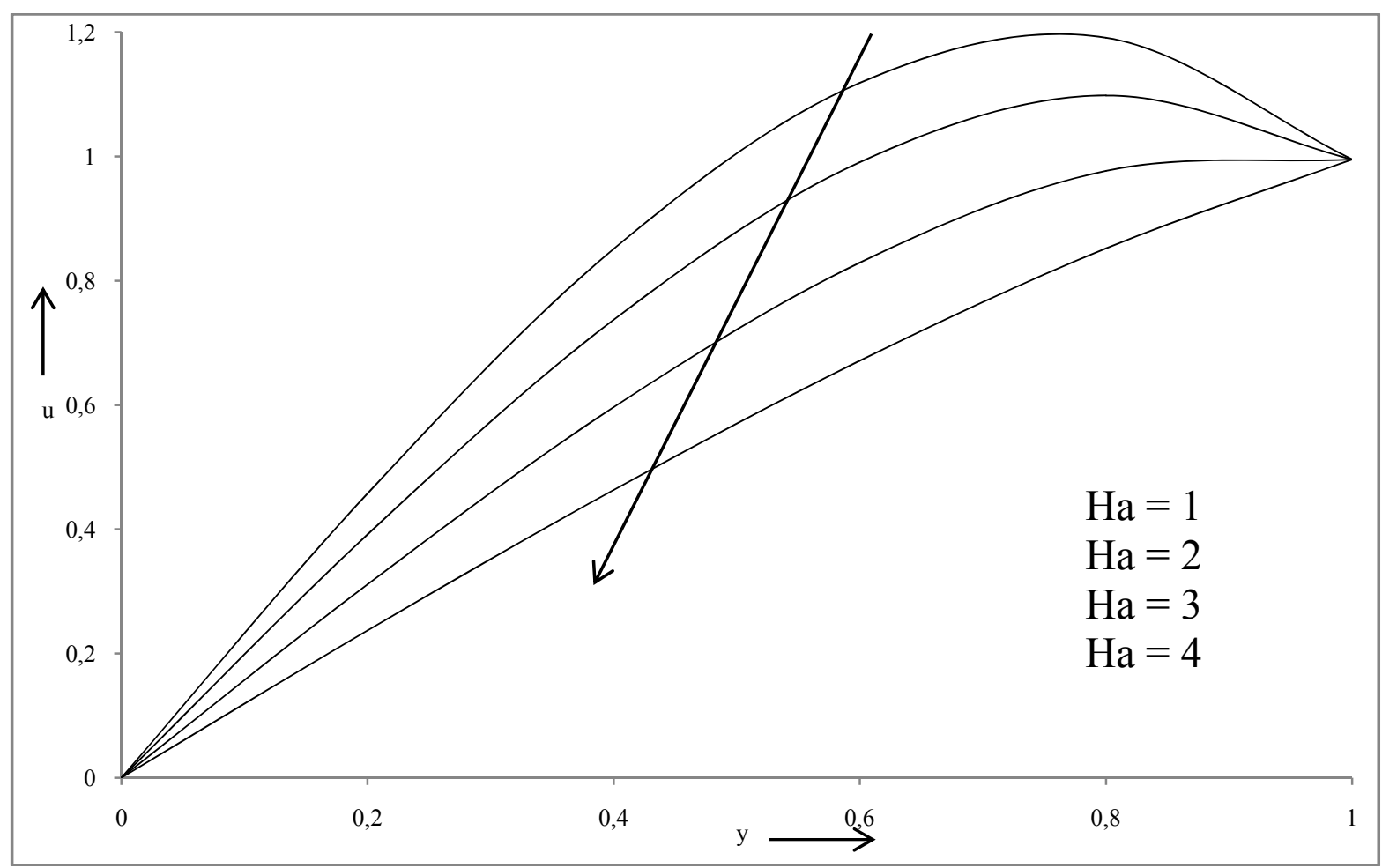

Fig.4. Velocity profiles versus $y$ for different values of $\mathrm{Ha}$ when $\mathrm{Gr}=10, \lambda=0.1, \mathrm{Re}=2, K=1, \mathrm{Pe}=0.71, R=2$, $S=5, \omega=1, \varphi=\pi / 4, g=0.1, t=0.1$.

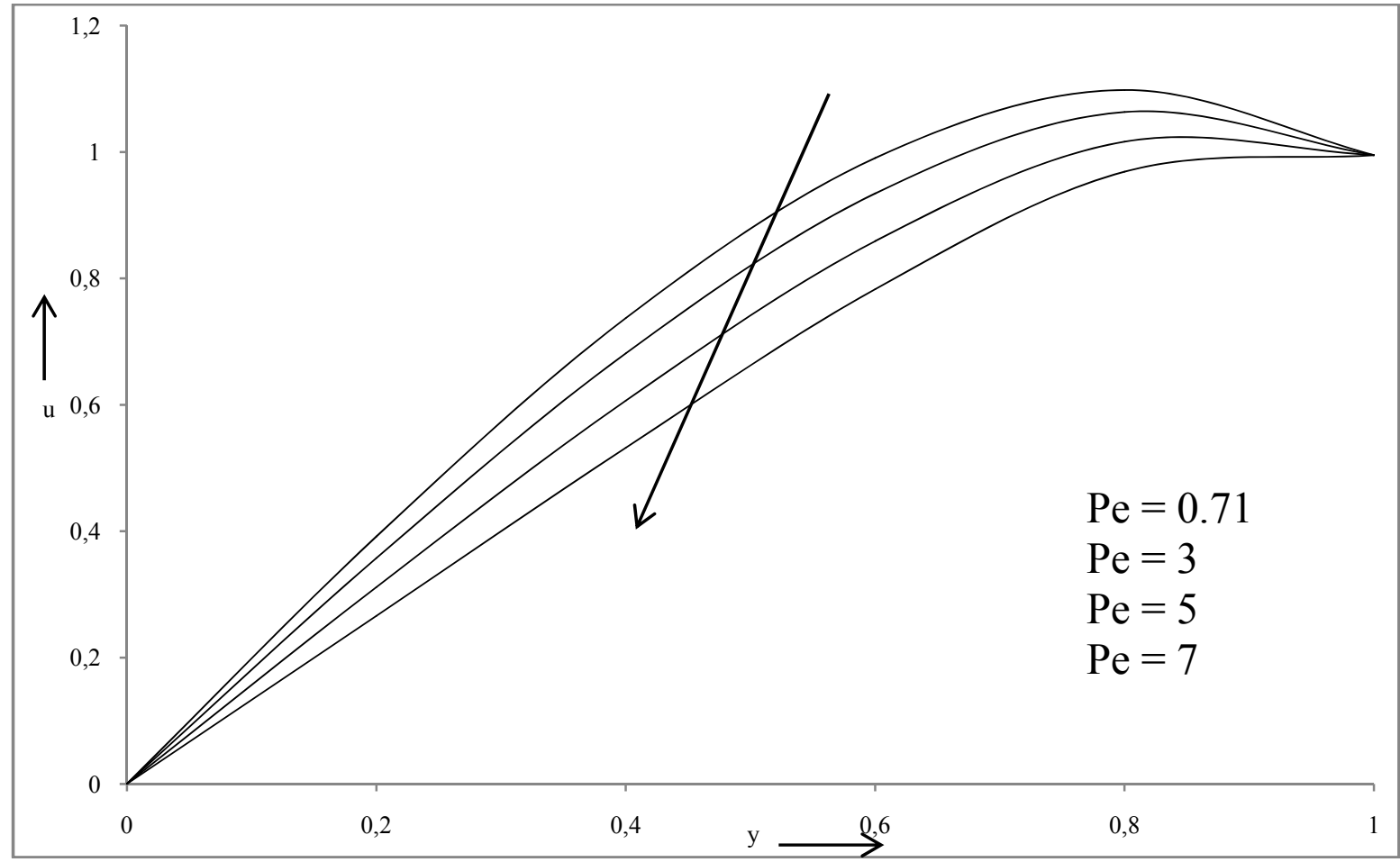

Fig.5. Velocity profiles versus $y$ for different values of $\mathrm{Pe}$ when $\mathrm{Gr}=10, \lambda=0.1, \mathrm{Re}=2, \mathrm{Ha}=2, K=1, R=2$, $S=5, \omega=1, \varphi=\pi / 4, g=0.1, t=0.1$. 


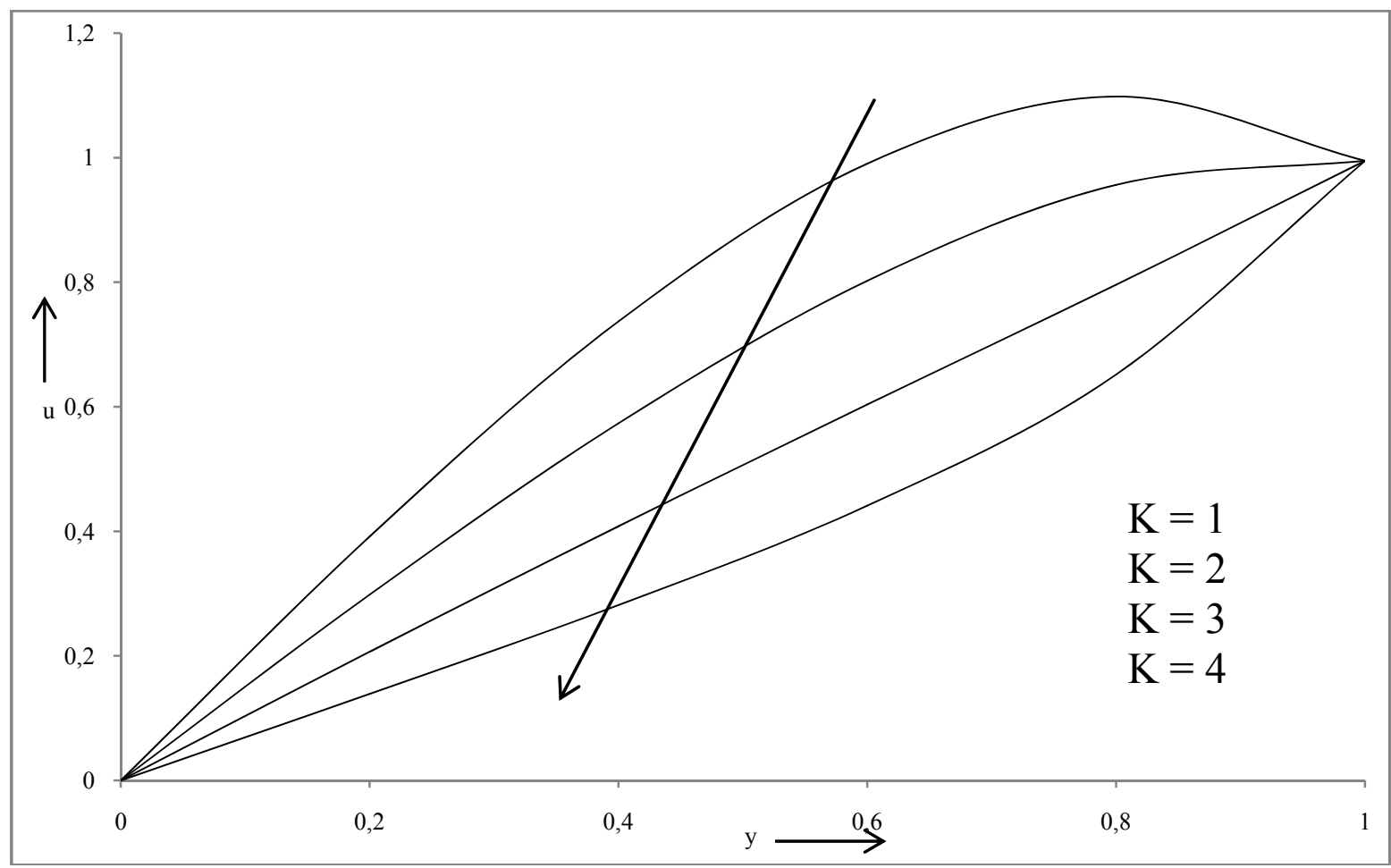

Fig.6. Velocity profiles versus $y$ for different values of $K$ when $\mathrm{Gr}=10, \lambda=0.1, \mathrm{Re}=2, \mathrm{Ha}=2, \mathrm{Pe}=0.71$, $R=2, S=5, \omega=1, \varphi=\pi / 4, g=0.1, t=0.1$.

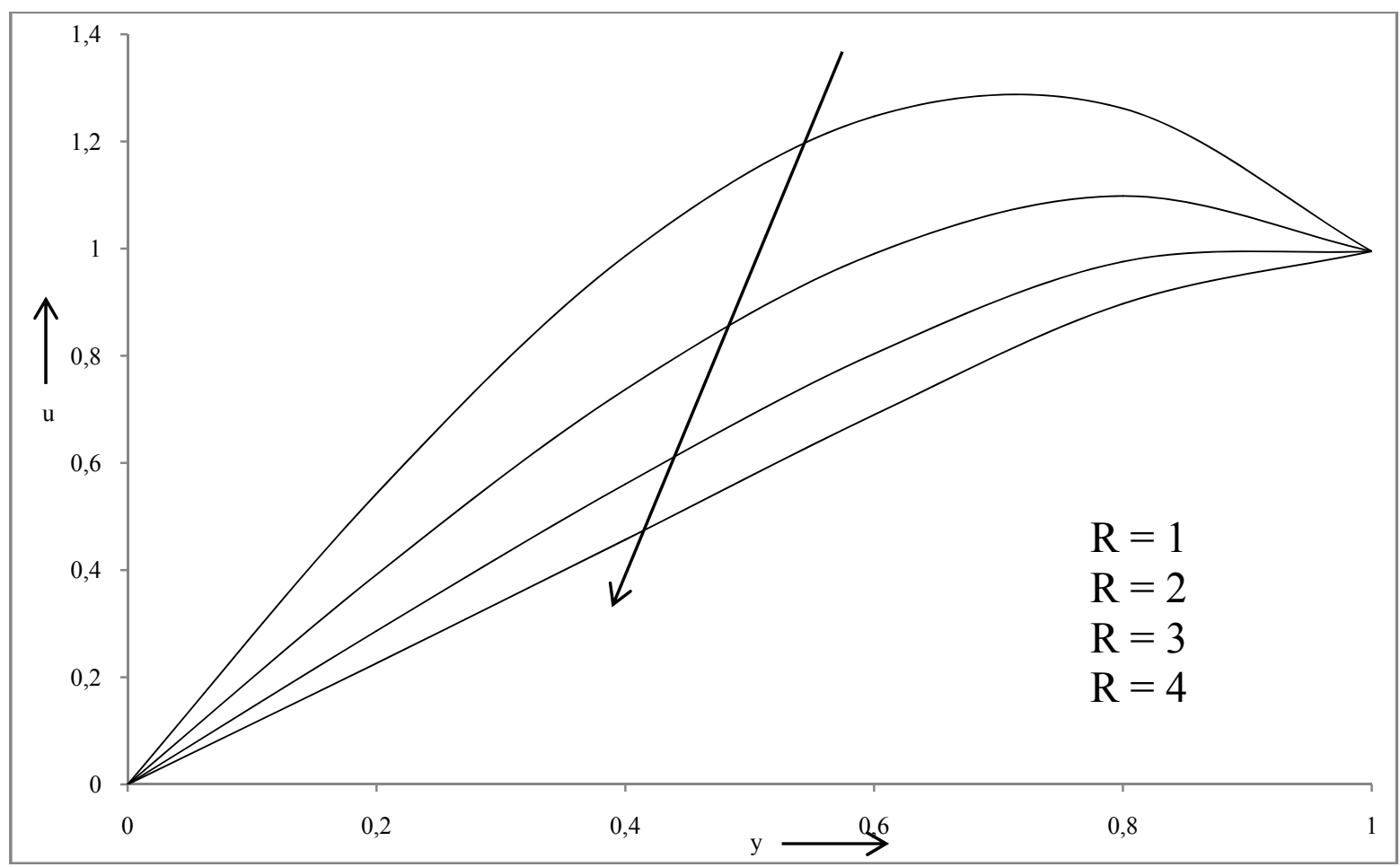

Fig.7. Velocity profiles versus $y$ for different values of $R$ when $\mathrm{Gr}=10, \lambda=0.1, \mathrm{Re}=2, \mathrm{Ha}=2, K=1$, $\mathrm{Pe}=0.71, S=5, \omega=1, \varphi=\pi / 4, g=0.1, t=0.1$. 


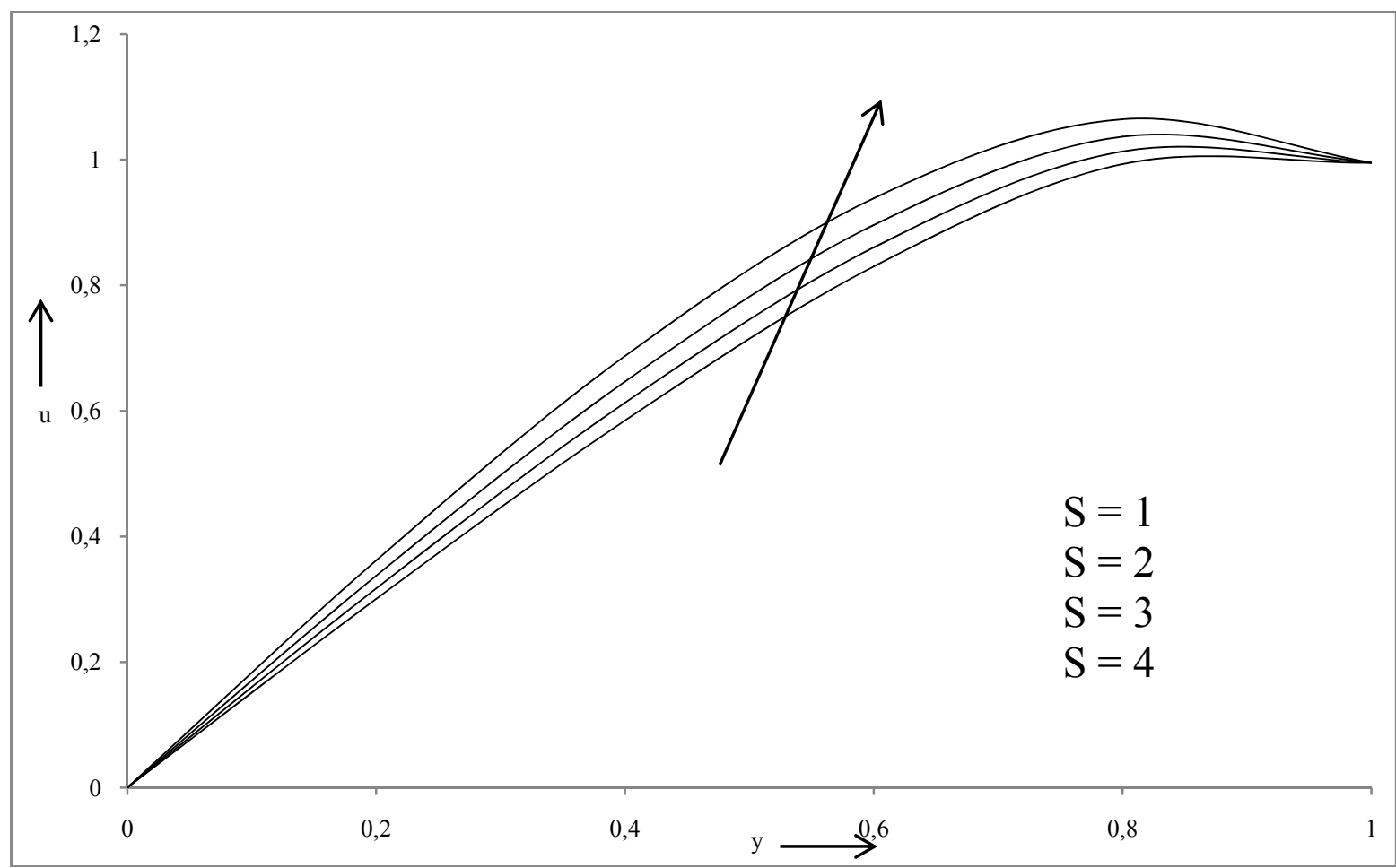

Fig.8. Velocity profiles versus $y$ for different values of $S$ when $\mathrm{Gr}=10, \lambda=0.1, \mathrm{Re}=2, \mathrm{Ha}=2, K=1$, $\mathrm{Pe}=0.71, R=2, \omega=1, \varphi=\pi / 4, g=0.1, t=0.1$.

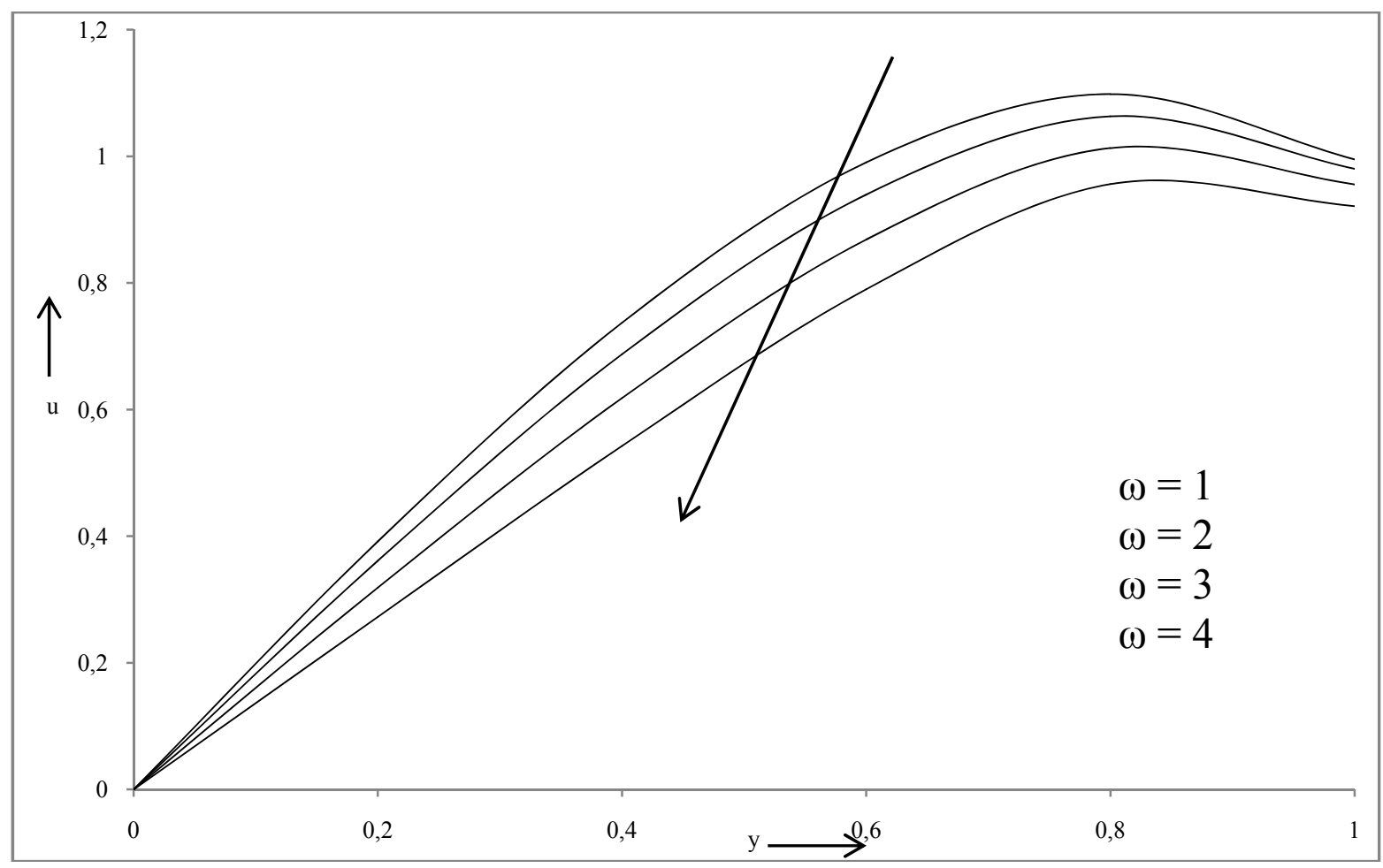

Fig.9. Velocity profiles versus $y$ for different values of $\omega$ when $\mathrm{Gr}=10, \lambda=0.1, \mathrm{Re}=2, \mathrm{Ha}=2, K=1$, $\mathrm{Pe}=0.71, R=2, S=5, \varphi=\pi / 4, g=0.1, t=0.1$. 


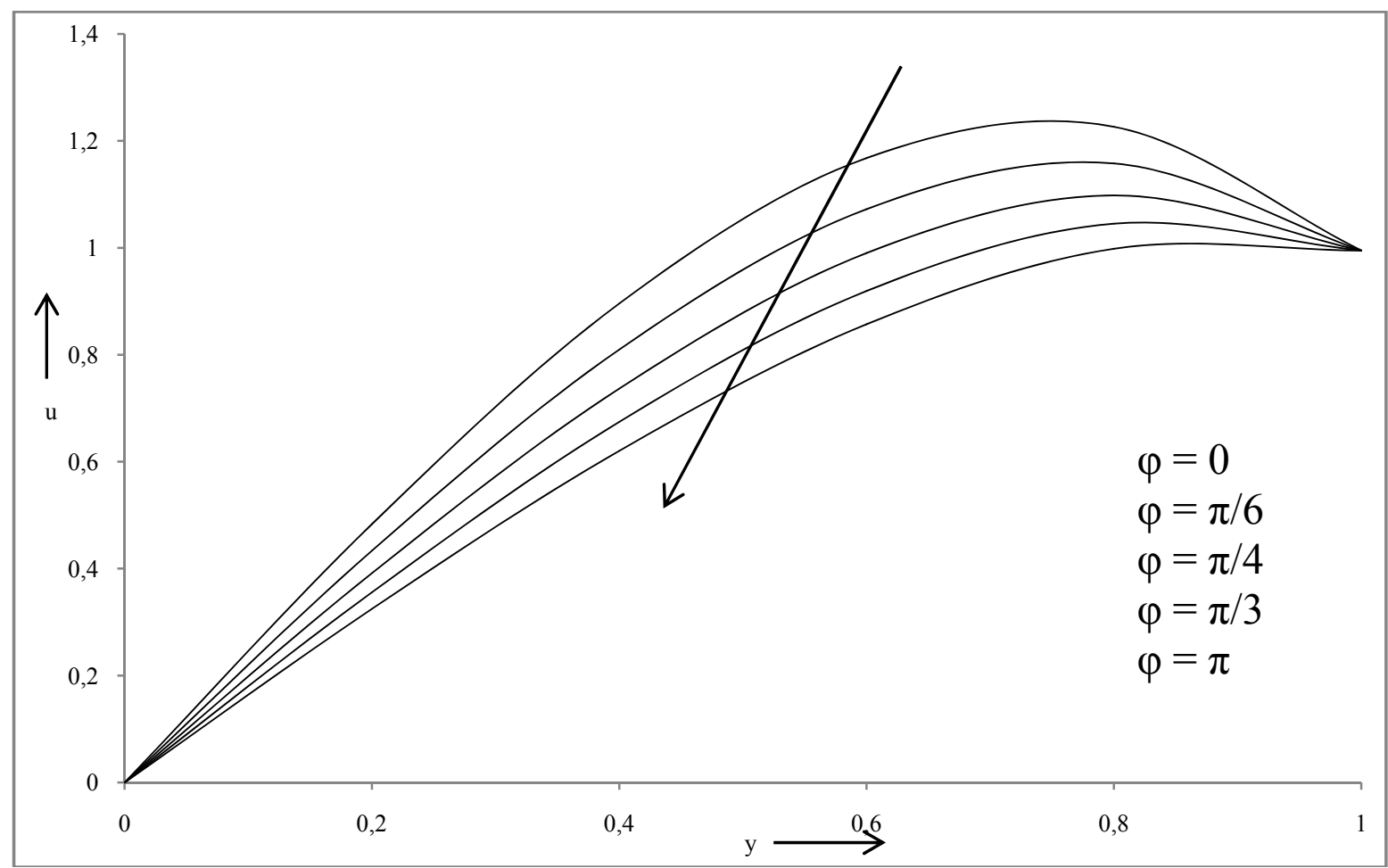

Fig.10. Velocity profiles versus $y$ for different values of $\varphi$ when $\mathrm{Gr}=10, \lambda=0.1, \mathrm{Re}=2, \mathrm{Ha}=2, K=1$, $\mathrm{Pe}=0.71, R=2, S=5, \omega=1, g=0.1, t=0.1$.

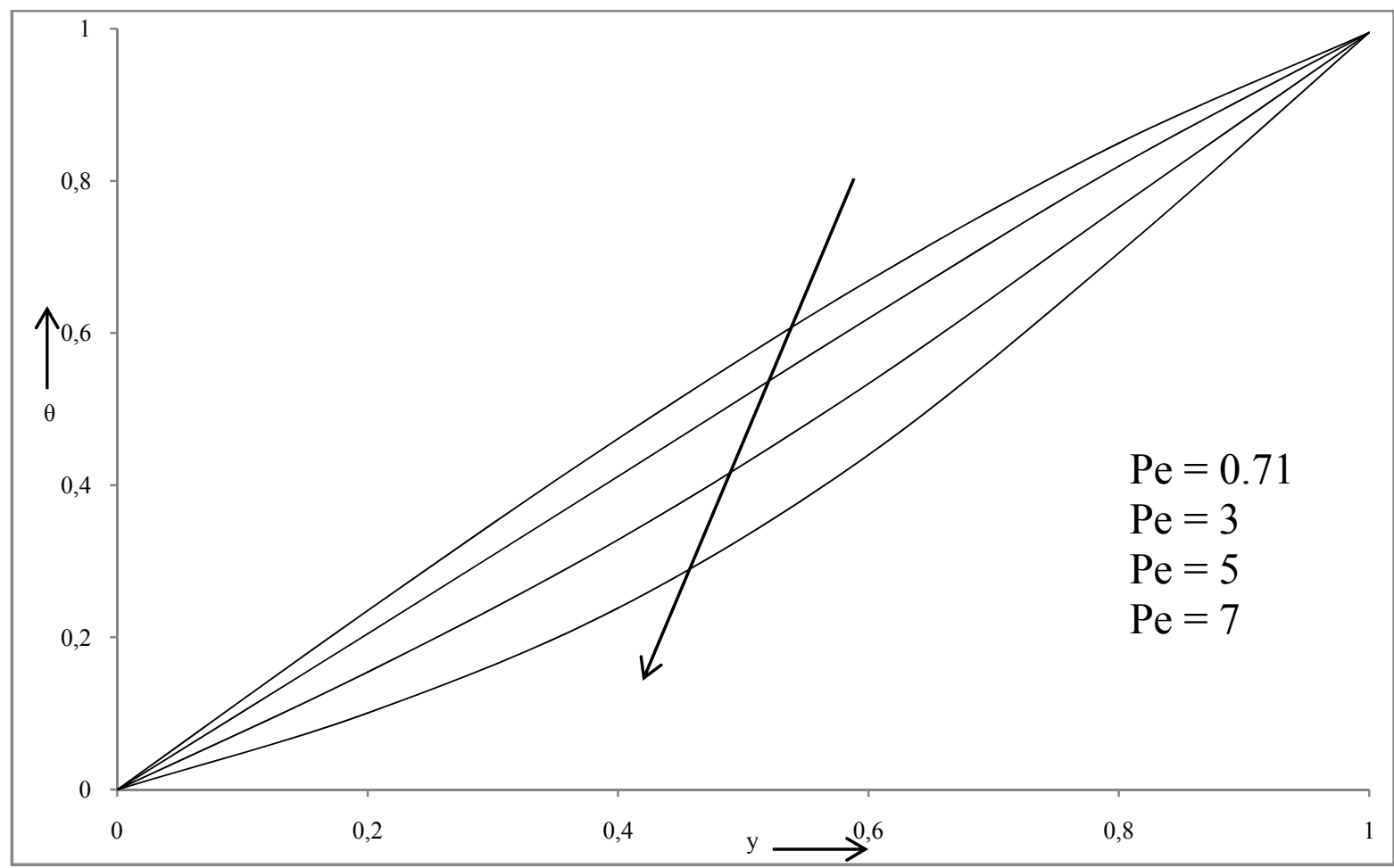

Fig.11. Temperature profiles versus $y$ for different values of $\mathrm{Pe}$ when $\mathrm{Gr}=10, \lambda=0.1, \mathrm{Re}=2, \mathrm{Ha}=2, K=1$, $R=2, S=5, \omega=1, \varphi=\pi / 4, g=0.1, t=0.1$. 


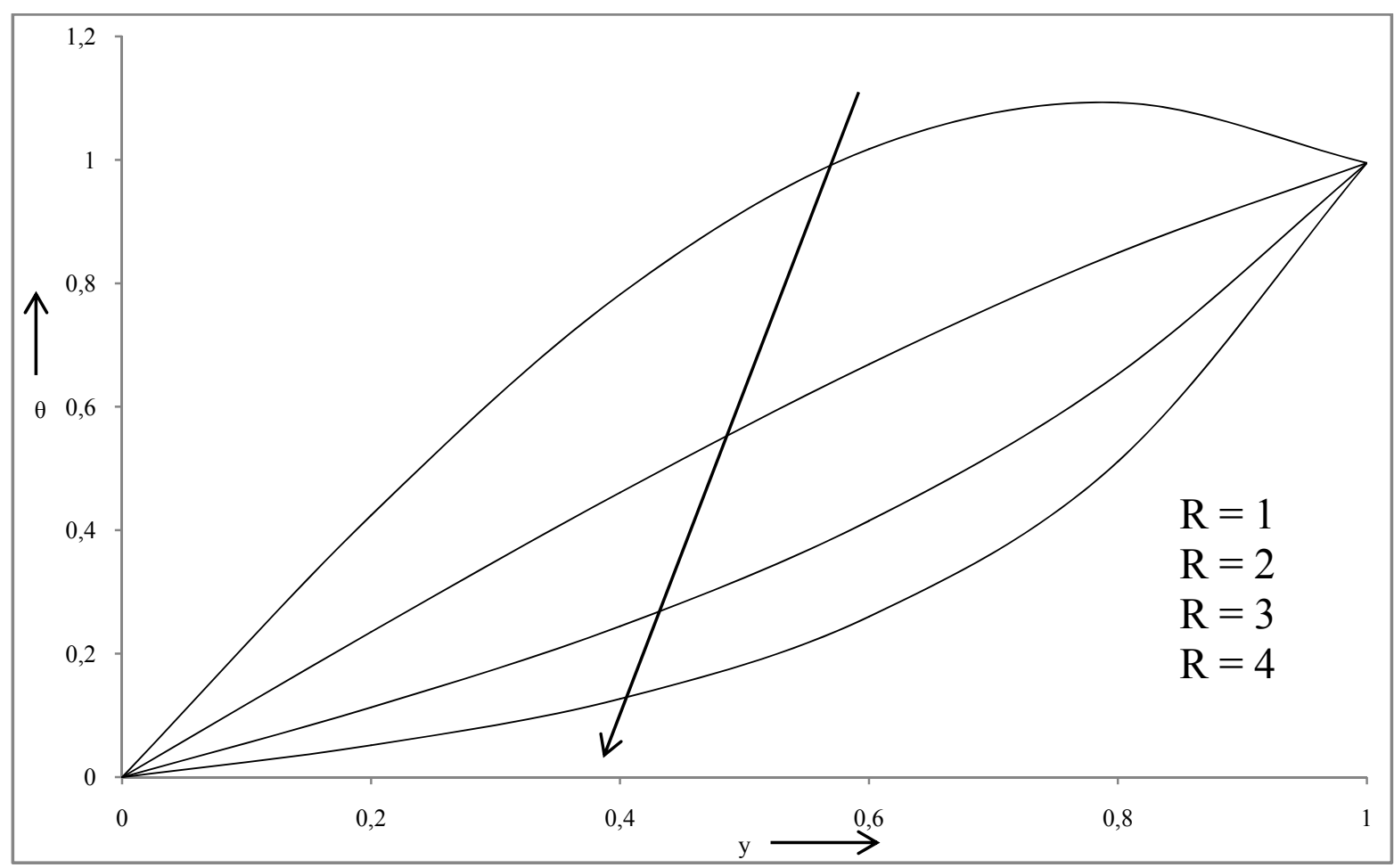

Fig.12. Temperature profiles versus $y$ for different values of $R$ when $\mathrm{Gr}=10, \lambda=0.1, \mathrm{Re}=2, \mathrm{Ha}=2, K=1$, $\mathrm{Pe}=0.71, S=5, \omega=1, \varphi=\pi / 4, g=0.1, t=0.1$.

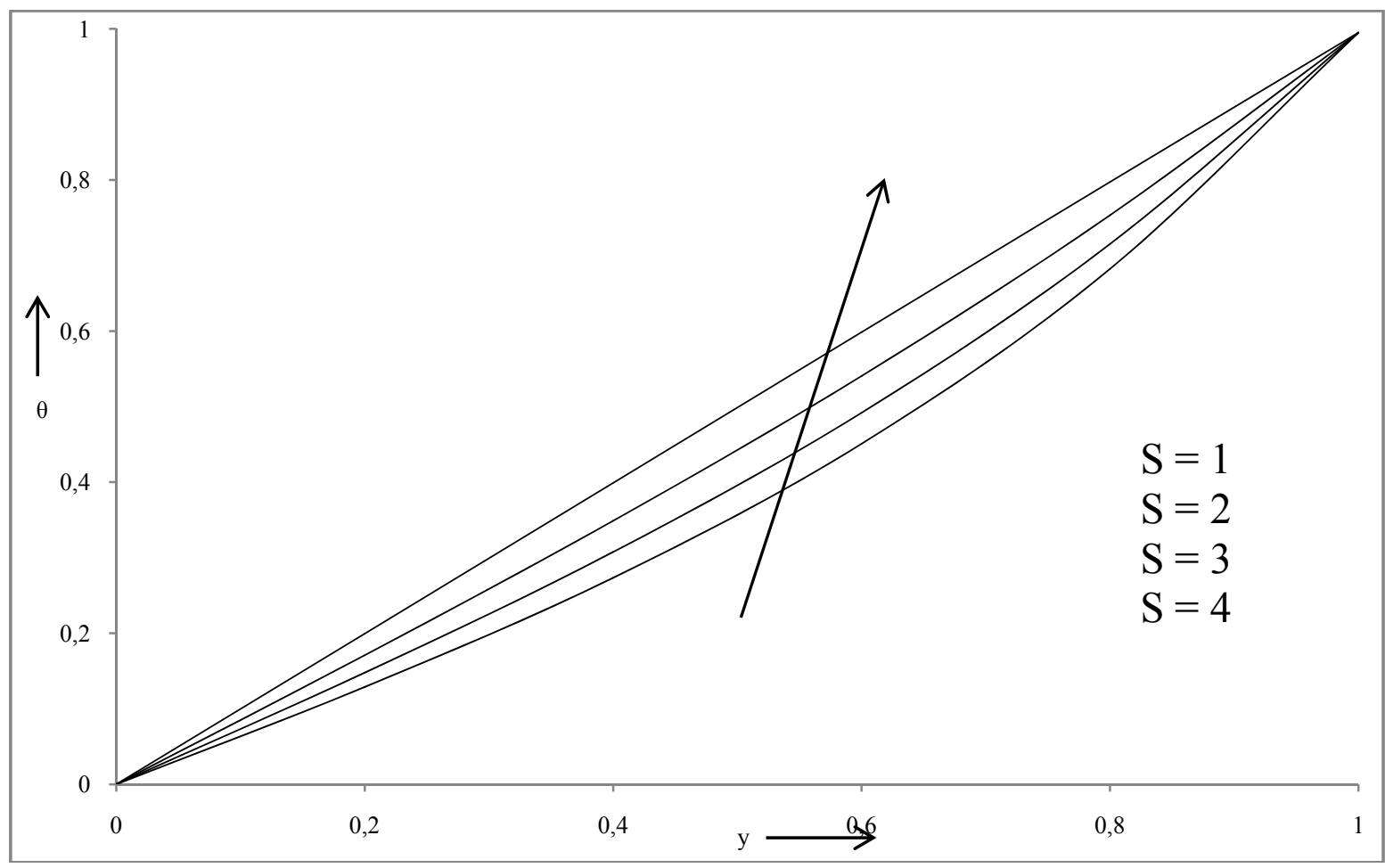

Fig.13. Temperature profiles versus $y$ for different values of $S$ when $\mathrm{Gr}=10, \lambda=0.1, \mathrm{Re}=2, \mathrm{Ha}=2, K=1$, $\mathrm{Pe}=0.71, R=2, \omega=1, \varphi=\pi / 4, g=0.1, t=0.1$. 


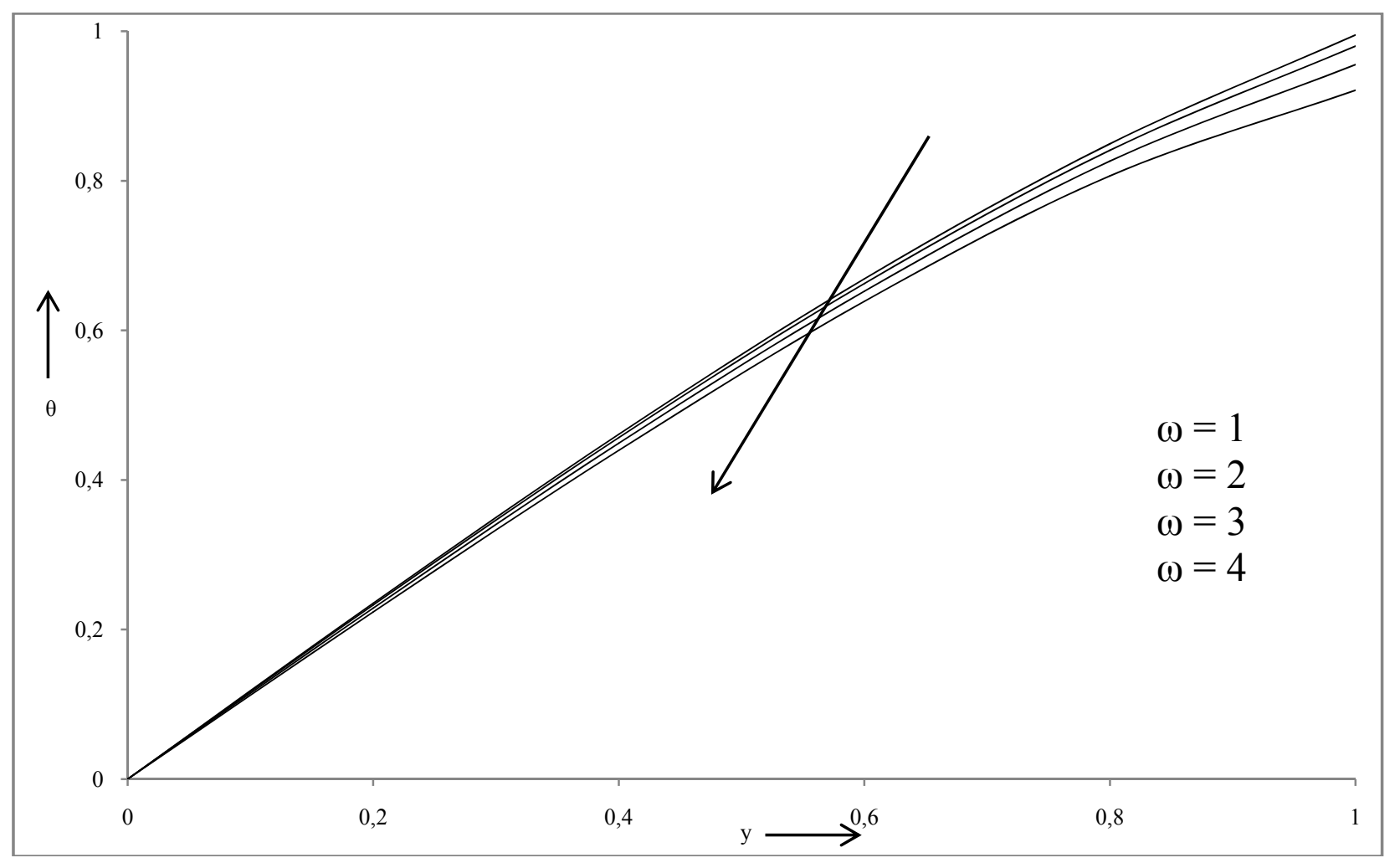

Fig.14. Temperature profiles versus $y$ for different values of $\omega$ when $\mathrm{Gr}=10, \lambda=0.1, \mathrm{Re}=2, \mathrm{Ha}=2, K=1$, $\mathrm{Pe}=0.71, R=2, S=5, \varphi=\pi / 4, g=0.1, t=0.1$.

It is observed from Tab. 1 that the skin-friction coefficient at the plate when $y=0$ increases as the values of Grashof number, heat source parameter or wave length increase, while it decreases due to increase in the values of Reynolds number, Hartmann number, Peclet number, permeability parameter, radiation parameter, frequency of the oscillation or angle between velocity and strength of the magnetic field. The skin-friction coefficient at the plate when $y=1$ increases due to increase in Reynolds number, Hartmann number, Peclet number, permeability parameter, radiation parameter, frequency of the oscillation or angle between velocity and strength of the magnetic field, while it decreases due to increase in the values of Grashof number, heat source parameter or wave length.

It is noted from Tab.2 that the Nusselt number at the plate when $y=0$ increases due to increase in Peclet number, radiation parameter or frequency of the oscillation, while it decreases due to increase in heat source parameter. The Nusselt number at the plate when $y=1$ increases with heat source parameter or frequency of the oscillation, while it decreases with the increase of Peclet number or radiation parameter. 
Table 1. Numerical values of skin friction coefficient at the plates for various values of physical parameters.

\begin{tabular}{|c|c|c|c|c|c|c|c|c|c|c|c|}
\hline $\mathrm{Gr}$ & $\lambda$ & $\mathrm{Re}$ & $\mathrm{Ha}$ & $K$ & $\mathrm{Pe}$ & $R$ & $S$ & $\omega$ & $\alpha$ & $C f_{0}$ & $C f_{1}$ \\
\hline 10 & 0.1 & 2 & 2 & 1 & 0.71 & 2 & 5 & 1 & $\pi / 4$ & 2.00269 & -1.1705 \\
\hline 1 & 0.1 & 2 & 2 & 1 & 0.71 & 2 & 5 & 1 & $\pi / 4$ & 0.78894 & 1.49301 \\
\hline 2 & 0.1 & 2 & 2 & 1 & 0.71 & 2 & 5 & 1 & $\pi / 4$ & 0.9238 & 1.19706 \\
\hline 10 & 0.2 & 2 & 2 & 1 & 0.71 & 2 & 5 & 1 & $\pi / 4$ & 2.04262 & -1.2105 \\
\hline 10 & 0.3 & 2 & 2 & 1 & 0.71 & 2 & 5 & 1 & $\pi / 4$ & 2.08254 & -1.2504 \\
\hline 10 & 0.1 & 1 & 2 & 1 & 0.71 & 2 & 5 & 1 & $\pi / 4$ & 2.04661 & -1.2057 \\
\hline 10 & 0.1 & 3 & 2 & 1 & 0.71 & 2 & 5 & 1 & $\pi / 4$ & 1.92593 & -1.097 \\
\hline 10 & 0.1 & 2 & 1 & 1 & 0.71 & 2 & 5 & 1 & $\pi / 4$ & 2.34739 & -1.7856 \\
\hline 10 & 0.1 & 2 & 3 & 1 & 0.71 & 2 & 5 & 1 & $\pi / 4$ & 1.58467 & -0.3293 \\
\hline 10 & 0.1 & 2 & 2 & 2 & 0.71 & 2 & 5 & 1 & $\pi / 4$ & 1.51808 & -0.1818 \\
\hline 10 & 0.1 & 2 & 2 & 3 & 0.71 & 2 & 5 & 1 & $\pi / 4$ & 1.04472 & 1.04248 \\
\hline 10 & 0.1 & 2 & 2 & 1 & 3 & 2 & 5 & 1 & $\pi / 4$ & 1.81904 & -0.9847 \\
\hline 10 & 0.1 & 2 & 2 & 1 & 7 & 2 & 5 & 1 & $\pi / 4$ & 1.33204 & -0.478 \\
\hline 10 & 0.1 & 2 & 2 & 1 & 0.71 & 1 & 5 & 1 & $\pi / 4$ & 2.80437 & -2.0602 \\
\hline 10 & 0.1 & 2 & 2 & 1 & 0.71 & 3 & 5 & 1 & $\pi / 4$ & 1.44633 & -0.493 \\
\hline 10 & 0.1 & 2 & 2 & 1 & 0.71 & 2 & 2 & 1 & $\pi / 4$ & 1.6103 & -0.7022 \\
\hline 10 & 0.1 & 2 & 2 & 1 & 0.71 & 2 & 3 & 1 & $\pi / 4$ & 1.7162 & -0.8322 \\
\hline 10 & 0.1 & 2 & 2 & 1 & 0.71 & 2 & 5 & 2 & $\pi / 4$ & 1.84024 & -1.0949 \\
\hline 10 & 0.1 & 2 & 2 & 1 & 0.71 & 2 & 5 & 3 & $\pi / 4$ & 1.61465 & -1.0127 \\
\hline 10 & 0.1 & 2 & 2 & 1 & 0.71 & 2 & 5 & 1 & $\pi / 6$ & 2.22249 & -1.5691 \\
\hline 10 & 0.1 & 2 & 2 & 1 & 0.71 & 2 & 5 & 1 & $\pi / 3$ & 1.81607 & -0.8107 \\
\hline
\end{tabular}

Table 2. Numerical values of Nusselt number at the plates for various values of physical parameters.

\begin{tabular}{|c|c|c|c|c|c|}
\hline $\mathrm{Pe}$ & $R$ & $S$ & $\omega$ & $\mathrm{Nu}_{0}$ & $\mathrm{Nu}_{1}$ \\
\hline 0.71 & 2 & 5 & 1 & -1.1839 & -0.6267 \\
\hline 3 & 2 & 5 & 1 & -1.0217 & -0.7748 \\
\hline 7 & 2 & 5 & 1 & -0.4706 & -1.374 \\
\hline 0.71 & 1 & 5 & 1 & -2.1778 & 0.91176 \\
\hline 0.71 & 3 & 5 & 1 & -0.5506 & -2.053 \\
\hline 0.71 & 2 & 2 & 1 & -0.7294 & -1.5719 \\
\hline 0.71 & 2 & 3 & 1 & -0.8489 & -1.2941 \\
\hline 0.71 & 2 & 5 & 2 & -1.1708 & -0.5805 \\
\hline
\end{tabular}

\section{Conclusions}

The effect of an inclined magnetic field on an oscillatory fluid flow between parallel plates in the presence of radiative heat flux and heat source is considered. The velocity and temperature profiles are obtained and shown through graphs. Dimensionless shear stress and rate of heat transfer at the plates are derived and their numerical values are presented through tables. In view of the above, the following conclusions are made: 
(i) The velocity of the fluid increases due to an increase in the Grashof number or wave length, while it decreases due to an increase in the Reynolds number, Hartmann number or permeability parameter.

(ii) The velocity and temperature of the fluid decrease due to an increase in the Peclet number, radiation parameter, heat source parameter or frequency of the oscillation.

(iii) As the Grashof number, heat source parameter or wave length increase, the skin-friction coefficient increases at the plate when $y=0$ and decreases at the plate when $y=1$.

(iv) An increase in the Reynolds number, Hartmann number, Peclet number, permeability parameter, radiation parameter, frequency of the oscillation or angle between velocity and strength of the magnetic field causes a decrease in the skin-friction coefficient at the plate when $y=0$ and an increase at the plate when $y=1$.

(v) As the Peclet number or radiation parameter increase, the Nusselt number increases at the plate when $y=0$ while it decreases at the plate when $y=1$.

(vi) Due to an increase in the frequency of the oscillation the Nusselt number increases at both the plates.

(vii) An increase in the heat source parameter leads to an increase in the Nusselt number at the plate when $y=1$ and a decrease at the plate when $y=0$.

\section{Nomenclature}

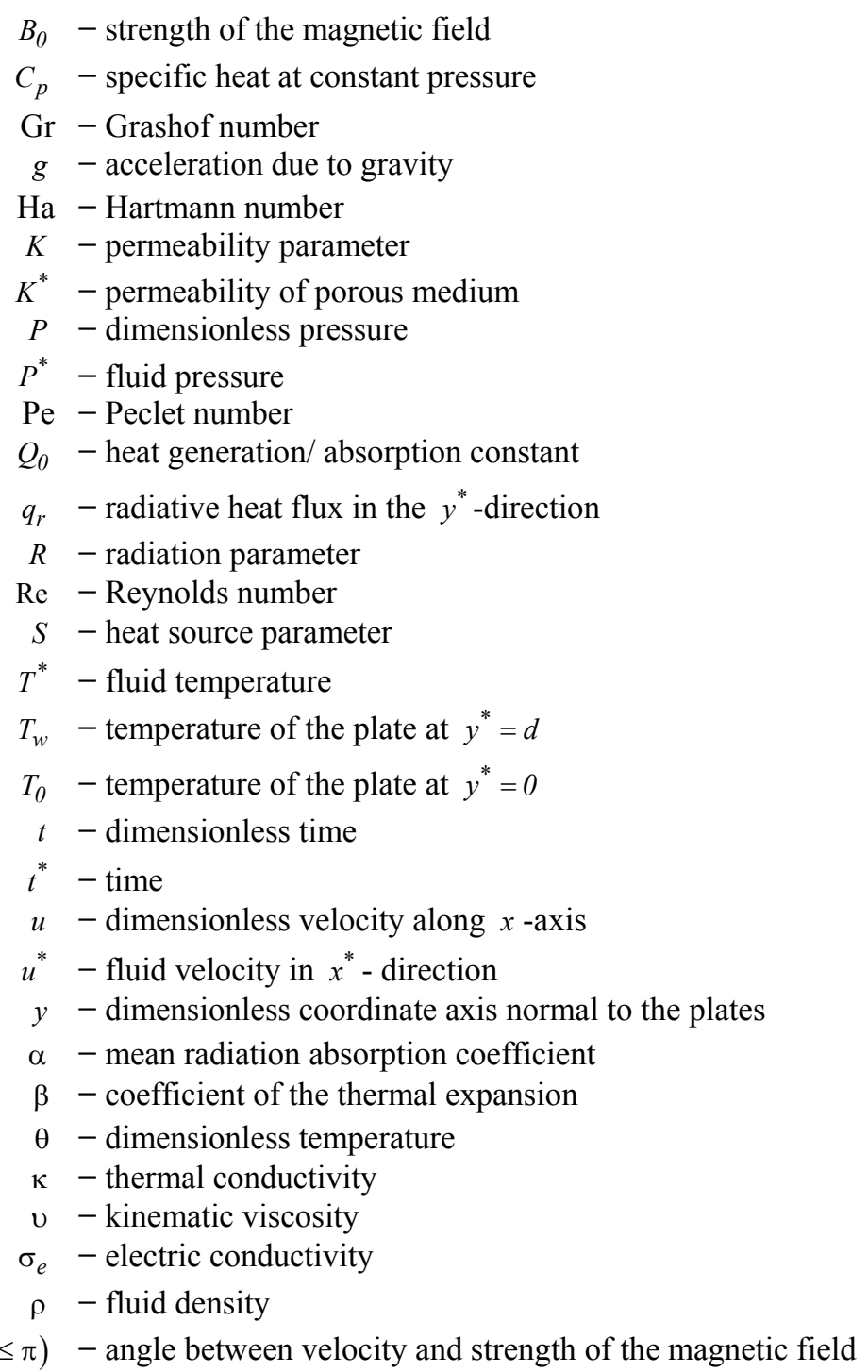




\section{References}

[1] Nigam S.D. and Singh S.N. (1960): Heat transfer by laminar flow between parallel plates under the action of transverse magnetic field. - Quarterly. J. Mech. Appl. Math., vol.13, pp.85-87.

[2] Verma P.D. and Bansal J.L. (1966): Flow of a viscous incompressible fluid between two parallel plates, one in uniform motion and the other at rest with uniform suction at the stationary plate. - Proc. Indian Acad. Sci., vol.64, pp.385-396.

[3] Soundalgekar VM. and Bhat J.P. (1971): Oscillatory channel flow and heat transfer. - Int. J. Pure Appl. Math., vol.15, pp.819-828.

[4] Raptis A., Massias C. and Tzivanidis G. (1982): MHD flow between two parallel plates with heat transfer. - Phys. Lett., vol.90, pp.288-289.

[5] Sharma P.R. and Sharma M.K. (1997): Unsteady flow and heat-transfer between two parallel plates. - Bull. Pure Appl. Science, India, vol.16, pp.183-198.

[6] Bhuyan B.C. and Hazarika G.C. (2001): Effect of magnetic field on pulsatile flow blood in a porous channel. Bio-Science Research Bulletin, vol.17, pp.05-112.

[7] Sharma P.R. and Chaturvedi R. (2003): Unsteady flow and heat transfer of an electrically conducting viscous incompressible fluid between two non-conducting parallel porous plates under uniform transverse magnetic field. - “Ganita Sandesh” J. Raj. Ganita Parishad, India, vol.17, pp.9-14.

[8] Ganesh S. and Krishnambal S. (2006): Magnetohydrodynamic flow of viscous fluid between two parallel porous plates. - J. of Appl. Sci., vol.6, pp.2450-2425.

[9] Ghosh S.K. (2007): Hydromagnetic fluctuating flow of a viscoelastic fluid in a porous channel. - J. of Applied Mechanics, vol.74, pp.177-180.

[10] Sharma P.R. and Singh G. (2008): Effects of variable thermal conductivity and heat source/sink on MHD flow near a stagnation point on a linearly stretching sheet. - J. of Appl. Fluid Mechanics, vol.2, pp.13-22.

[11] Makinde O.D. and Aziz A. (2010): MHD mixed convection from a vertical plate embedded in a porous medium with a convective boundary condition. - Int. J. of Thermal Sci., vol.49, pp.1813-1820.

[12] Adhikary S.D. and Misra J.C. (2011): Unsteady two-dimensional hydromagnetic flow and heat transfer of a fluid. - Int. J. of Applied Math. and Mech., vol.7, pp.1-20.

[13] Singh K.D. (2012): Viscoelastic mixed convection MHD oscillatory flow through a porous medium filled in a vertical channel. - Int. J. of Phy. and Math. Sci., vol.3, pp.194-205.

[14] Das U.J. (2013): Viscoelastic effects on unsteady two dimensional heat and mass transfer of a viscoelastic fluid in a porous channel with radiative heat transfer. - Engg. J. of Scientific Reasearch, vol.5, pp.67-72.

[15] Sandeep N. and Sugunamma V. (2013): Effect of an inclined magnetic field on unsteady free convection flow of a dusty viscous fluid between two infinite flat plates filled by a porous medium. - Int. J. of Appl. Maths. Modelling, vol.1, pp.16-33.

[16] Joseph K.M., Daniel S. and Joseph G.M. (2014): Unsteady MHD Couette flow between two infinite parallel porous plates in an inclined magnetic field with heat transfer. - Int. J. of Mathematics and Statistics Invention, vol.2, pp.103-110.

[17] Cogley A.C., Vincenti W.G. and Gill S.E. (1968): Differential approximation for radiative transfer in a non-gray gas near equilibrium. - AIAA J., vol.6, pp.551-553. 